\title{
Valoración de la implantación del seguimiento ecográfico del acceso vascular autólogo
}

\author{
María del Valle Garrido López, Carolina Sesmero Ramos, Almudena Ortigosa Barriola, Enrique Gruss Vergara \\ Hospital Universitario Fundación Alcorcón. Madrid. España
}

\section{Resumen}

Un acceso vascular adecuado es imprescindible para el tratamiento renal sustitutivo mediante hemodiálisis. Las enfermeras participan de forma fundamental en el seguimiento del mismo. El acceso vascular autólogo es considerado el acceso vascular de elección, pero también es el que más complicaciones iniciales presenta.

El objetivo de este trabajo es valorar la eficacia del ecógrafo para el seguimiento y punción de estos accesos vasculares.

Material y Métodos: Estudio observacional retrospectivo de octubre 2014 a febrero 2015. Se incluyeron 53 pacientes con fístula autóloga. 31 con acceso vascular en uso y 22 con acceso de nueva creación.

Se inició el uso del ecógrafo previa formación específica en ecografía doppler de tres enfermeras y un nefrólogo.

Se realizaron 73 ecografías en las que se determinaron flujo arterial, diámetro y profundidad venosa, anomalías anatómicas y disfunciones.

Resultados: Se identificaron 9 accesos autólogos de nueva creación con flujos arteriales por debajo de parámetros de normalidad. Se asociaron a accesos vasculares menores de 3 meses, flujos menores de $500 \mathrm{ml} / \mathrm{min}$. De 15 estenosis identificadas hubo un mayor porcentaje en accesos radiocefálicos., también se confirmaron 2 trombosis completas y 1 parcial.

Correspondencia:

María del Valle Garrido López

Unidad de Diálisis

Hospital Universitario Fundación de Alcorcón

Calle Budapest, s/n. 28922 Alcorcón. Madrid

Email: mvagarrido@yahoo.es
Se realizaron 9 punciones ecoasistidas en fístulas autólogas de nueva creación y se modificaron zonas de punción habituales en 8 pacientes.

Conclusiones: El ecógrafo ha demostrado ser un instrumento útil para facilitar las punciones y seguimiento del acceso vascular. Permite identificar nuevas zonas de punción.

Permite una valoración objetiva de los AV autólogos mediante parámetros ecográficos favoreciendo su seguimiento y alertando de forma temprana sobre posibles disfunciones, posibilitando el tratamiento precoz de éstas.

Permite establecer protocolos de seguimiento de acceso vascular más estrecho en sus tres primeros meses de vida y de forma estandarizada a lo largo de la vida del AV.

PALABRAS CLAVE

- HEMODIÁLISIS

- ACCESO VACULAR AUTÓLOGO

- PUNCIÓN

- ECOGRAFÍA

Assessment to the implementation of an ultrasound monitoring of autologous vascular access

\section{Abstract}

An adequate vascular access is essential for renal replacement therapy by hemodialysis. Nurses participate in the monitoring of this fundamentally. Autologous vascular access is considered the preferred vascular access, but also the one with more initial complications. 
The aim of this study is to evaluate the efficacy of ultrasound for monitoring and puncture of this vascular access.

Methods: A retrospective observational study from October 2014 to February 2015 was carried out. Fifty-three patients with autologous fistula were included: 31 already in use and 22 newly implanted vascular access.

The use of ultrasound scanner was initiated prior specific training to three nurses and a nephrologist at Doppler echography.

Seventy-three ultrasound scans which determined blood flow, venous diameter and depth, anatomical abnormalities and dysfunctions were carried out.

Results: Nine newly-implanted autologous vascular accesses with arterial flows below normal parameters were identified. Puncture sites were modified in $8 \mathrm{ca}-$ ses. 9 ultrasound assisted punctures were performed in newly-implanted autologous fistulas. Fifteen stenosis were identified. Two full and one partial thrombosis were confirmed. An association between vascular accesses (VA) younger than 3 months and flows below $500 \mathrm{ml} / \mathrm{min}$ was found. Increased percentage of stenosis in radiocephalic VA was identified.

Conclusions: The ultrasound scanner has proven to be a useful tool to facilitate punctures and monitoring of vascular access. It identifies new areas of puncture and allows an objective evaluation of autologous AV by ultrasound parameters favoring its monitoring and alerting on possible malfunctions early, allowing early treatment of these.

This allows establishing protocols for monitoring in narrow VA during its first three months and in a standardized manner throughout the life of the VA.

\section{KEYWORDS}

- HEMODIALYSIS

- AUTOLOGOUS VASCULAR ACCESS

- PUNCTURE

- ULTRASOUND

\section{Introducción}

Un buen funcionamiento del acceso vascular (AV) es un requisito indispensable para el tratamiento renal sustitutivo mediante hemodiálisis (HD) $)^{1-4}$ y por tanto, de vital importancia para el paciente influyendo en su calidad de vida $^{3-5}$. Las enfermeras participan de forma fundamental en el mantenimiento de la supervivencia y permeabilidad del mismo 2,3,5.

Entre los diversos tipos de AV, el autólogo o nativo está considerado como el mejor, por ser el que menos complicaciones presenta y el que se mantiene más tiempo permeable ${ }^{3,6}$. Sin embargo, inicialmente es el tipo de AV que más trombosis presenta y el más difícil de canali$\operatorname{zar}^{1,7}$.

La ecografía puede ser un instrumento útil para la valoración, manejo y seguimiento de los accesos vasculares autólogos ${ }^{1,7}$.

Se ha justificado la ecografía como método diagnóstico en diversas publicaciones ${ }^{1,7}$. En los AV autólogos de nueva creación los parámetros ecográficos de AV maduro para inicio de punciones son: diámetros de vena eferente $>0.4-0.5 \mathrm{~cm}$, profundidad $<0.6 \mathrm{~cm}$ y flujo vascular (Qa) $>500 \mathrm{ml} / \mathrm{min}^{1}$. De esta manera se obtienen parámetros objetivos para determinar si el AV es un acceso maduro para el inicio de las punciones. Hasta ahora, la valoración del grado de maduración de una FAVI que realizaba la enfermera se basaba en la exploración física y en criterios clínicos.

En el caso de accesos maduros en uso, es la enfermera la que vive el día a día del paciente, y experimenta en primera persona las posibles dificultades en el uso del acceso vascular. Es la enfermera la que da la voz de alarma cuando aparece una posible disfunción en el acceso vascular $2,3,5,6,7,8$. Cuando esto sucede, se recomienda la ecografía como exploración de imagen de primera elección ${ }^{13,5,7,9}$ en manos de un explorador experimentado, sin necesidad de fistulografía confirmatoria, realizándola sólo en casos en los que la ecografía no sea concluyen$\mathrm{te}^{1,9}$.

\section{Objetivo general}

Valorar la eficacia del uso del ecógrafo para el manejo del acceso vascular autólogo. 


\section{Objetivos específicos}

Evaluar la utilidad de la ecografía doppler para valorar la maduración del acceso vascular autólogo de nueva creación.

Evaluar la incidencia de modificación de zonas de punción en AV en uso.

Evaluar la eficiencia de las punciones realizadas con técnica ecoguiada y ecoasistida.

Evaluar la utilidad del ecógrafo para identificar la disfunción de acceso vascular autólogo de forma temprana.

\section{Material y Métodos}

Se estudiaron los pacientes de una unidad de diálisis hospitalaria nivel 2 de la Comunidad de Madrid que cumplieron los siguientes criterios de inclusión:

Pacientes con acceso vascular autólogo:

- De nueva creación procedente de consulta ERCA.

- De nueva creación en programa de HD que se dializaban a través de catéter.

- Pacientes con acceso vascular autólogo en uso.

Se realizó un estudio observacional retrospectivo desde octubre 2014 a abril 2015.

Se consideraron las siguientes variables:

- Maduración del AV autólogo de nueva creación a las cuatro semanas de creación, previamente a iniciar las punciones. Se valoró: diámetro y profundidad venosa y flujo arterial del acceso.

- Número de cambios de zona de punción en AV autólogos en uso.

- Número de punciones exitosas realizadas con ayuda del ecógrafo.

- Número de disfunciones identificadas.

Se analizó la asociación entre variables:

- Estenosis detectadas con tipo de AV/edad/sexo.

- Flujo AV medido con eco doppler con tipo de AV/edad/ sexo.

Para la puesta en marcha de este proyecto se contó con un ecógrafo doppler. Tres enfermeras y un nefrólogo se formaron en su manejo.
Se establecieron unos tiempos de actividad de enfermera dedicados a las ecografías. En la realización de la técnica preferentemente estuvieron presentes enfermera y nefrólogo, siendo ambos quienes realizaban el estudio. Si no podían estar los dos era realizada por uno de ellos. Si se detectaba alguna posible disfunción o alteración en el acceso estudiado, se comunicaba al nefrólogo.

En pacientes en consulta ERCA y pacientes en HD con catéter con nuevo acceso vascular autólogo, se realizó ecografía de éstos, tras cuatro semanas desde su creación aproximadamente y previamente a la primera punción.

En pacientes en programa de HD con AV autólogo en uso, se programaron ecografías seriadas cada seis meses y en aquellos con sospecha de disfunción cada tres meses. El estudio ecográfico se realizó previamente a la sesión de hemodiálisis o en un día de no diálisis.

Todas las exploraciones realizadas por la enfermera quedan registradas e informadas en la historia clínica electrónica del paciente, como nota de enfermería sobre estudio ecográfico del acceso vascular. En este apartado se incluyeron: tipo de acceso, diámetro y profundidad venoso, flujo arterial, si aparecieron anomalías anatómicas y se recomendaron zonas de punción en accesos de nueva creación y zonas de punción alternativas en $\mathrm{AV}$ en uso.

Para el manejo estadístico de estas variables se creó una base de datos excell, que se transportó al paquete estadístico SPSS.

\section{Resultados}

Se realizaron un total de 73 ecografías a 53 pacientes, 38 hombres y 15 mujeres, y la edad media fue $67 \pm 13$ años (29-88). El 56\% pacientes se encontraban en programa de HD y el resto en seguimiento en consulta de ERCA. Se realizaron ecografías a 22 accesos de nueva creación y a 31 accesos en uso. La distribución según tipo de acceso aparece en la Tabla 1.

El programa de seguimiento ecográfico permitió:

- Identificar 9 accesos autólogos de nueva creación (41 $\%$ ) con flujo arterial inferior al establecido como criterio de maduración ( $500 \mathrm{ml} / \mathrm{min}$ ).

- Describir una asociación entre AV menores de 3 meses de vida y flujo AV inferior a $500 \mathrm{ml} / \mathrm{min}$. 
Tabla 1. Descripción de AV autólogo según localización.

\begin{tabular}{|l|c|c|c|c|c|c|}
\hline Tipo AV & N & $\%$ & $\begin{array}{c}\text { Edad } \\
\text { AV (meses) } \pm \text { ds }\end{array}$ & $\begin{array}{c}\text { \% estenosis } \\
\text { Según localización }\end{array}$ & $\begin{array}{c}\text { \% estenosis } \\
\text { del total de AV }\end{array}$ \\
\hline$<00$ ml/min. \\
\hline Radio cefálicas (RC) & 26 & 49,06 & $28,77 \pm 42.65$ & $38,46 \%$ & $18,87 \%$ & $23,1 \%$ \\
\hline Húmero cefálicas (HC) & 19 & 35,85 & $56,89 \pm 70,98$ & $26,32 \%$ & $9,43 \%$ & $5.3 \%$ \\
\hline Húmero basílicas (HB) & 6 & 11,32 & $14,17 \quad 16,93$ & $0,00 \%$ & $0,00 \%$ & $16,7 \%$ \\
\hline Húmero perforantes (HP) & 2 & 3,77 & $7,005,66$ & $0,00 \%$ & $0,00 \%$ & $50 \%$ \\
\hline
\end{tabular}

- Modificar atendiendo a criterios ecográficos, las zonas de punción en 8 casos. (25\% de los accesos autólogos en uso).

- Realizar punciones ecoasistidas en todos los AV autólogos de nueva creación. En 2 casos estas punciones fueron fallidas, coincidiendo en ambos casos con bajo flujo del acceso y escaso desarrollo.

- Detectar 15 estenosis hasta el momento desconocidas (28.3\%). 3 de ellas significativas (20\%) en las cuales se procedió al tratamiento según criterio médico y 12 no significativas $(20 \%)$, que se mantienen en seguimiento ecográfico estrecho.

- Confirmar 2 trombosis de AV completas y 1 parcial.

La distribución de estenosis y vida (meses) de AV aparece reflejada en el siguiente gráfico.



Gráfico 1. Porcentaje de estenosis según vida (meses) de AV autólogo.

\section{Discusión}

El uso del ecógrafo por parte de la enfermera nos ha permitido valorar la maduración de los AV de nueva creación antes de iniciar las punciones. Mediante la valoración ecográfica de la maduración del AV evitamos punciones precoces en accesos inmaduros y podemos determinar aquellos que no se van a desarrollar o por el momento no cumplen los criterios para comenzar a puncionar. Este resultado está en concordancia con estudios previos que apoyan la ecografía como método evaluador de AV maduro $1,6,8$.

Encontramos además asociación entre AV menores de 3 meses de vida y flujos AV menores de $500 \mathrm{ml} / \mathrm{min}$. Este hallazgo apoya la necesidad de realizar un seguimiento estrecho de los AV en los tres primeros meses de vida. No obstante nuestros datos no permiten identificar como se comportan los $\mathrm{AV}$ de larga vida.

Al igual que otros autores ${ }^{5}$ hemos conseguido identificar nuevas zonas de punción en pacientes en programa de HD crónica aumentando así territorio venoso puncionable complementando métodos tradicionales de valoración como son la palpación y auscultación. Esto nos permite cambiar la técnica de punción en algunos accesos de "punción en área" a "punción escalonada", con los beneficios que eso supone.

Las punciones dificultosas suponen un elemento de estrés importante tanto de los pacientes como de los enfermeros de hemodiálisis. En línea con los resultados de Pons, Hernández López y López González, nuestro estudio confirma que la punción ecoguiada y ecoasistida facilita las punciones en $\mathrm{AV}$ autólogos y disminuyen el número de punciones fallidas. A pesar de los excelentes resultados, hasta la fecha, los datos son insuficientes para afirmar que mejore la supervivencia del $\mathrm{AV}^{1,10}$.

Hemos realizado una valoración objetiva de AV autólogos obteniendo parámetros ecográficos que nos permitirán realizar el seguimiento de estos a lo largo de su vida. Hallamos mayor número de estenosis en AV radiocefálicos, sin embargo no alcanza la significación estadística. No encontramos asociación entre estenosis y la vida del AV. Dicho hallazgo obliga a realizar un seguimiento estrecho y protocolizado a lo largo de toda la vida del $\mathrm{AV}^{1,5,7,9}$. 
Nuestro objetivo fue realizar una evaluación piloto del potencial de la ecografía en la unidad de hemodiálisis. Por ello una de las limitaciones de nuestro estudio es el número limitado de sujetos incluidos en el mismo. Otra limitación es que la técnica ecográfica es operador-dependiente, lo que significa que la práctica y la experiencia del profesional que maneja este método es muy importante para la obtención de resultados fiables. Es fundamental el aprendizaje y experiencia previa 6 . Por otra parte es innegable que la ecografía supone un coste inicial (recursos y formación), si bien puede justificarse por la disminución de fistulografías necesarias una vez implantado el seguimiento ecográfico de $\mathrm{AV}^{1}$.

Concluimos que el uso del ecógrafo permite valorar objetivamente el desarrollo en AV de nueva creación previamente a comenzar las punciones, permite realizar seguimiento AV autólogo maduro identificando nuevas zonas de punción y disfunciones y constituye un método innovador para ayudarnos en nuestro trabajo diario. La técnica ecográfica aumenta el campo de intervenciones y competencias de la enfermería, abriéndonos una nueva área de formación.

Aunque nuestra experiencia es corta, los resultados hasta ahora han sido positivos. Así mismo, creemos necesaria la ampliación del estudio incluyendo nuevos pacientes y realizando un seguimiento a largo plazo de estos AV autólogos.

\section{Recibido: 11 julio 2015}

Revisado: 9 septiembre 2015

Modificado: 3 noviembre 2015

Aceptado: 16 noviembre 2015

\section{Bibliografía}

1. Ibeas López J., Vallespin Aguado J. Ecografía del acceso vascular para hemodiálisis: conceptos teóricos y prácticos. Criterios. Nefrología sup. Ext. 2012;3(6):21-35.

2. Hernández López J., Mayor Iturburuaga J.M. La enfermería de diálisis con la ecografía en los accesos vasculares para hemodiálisis. Caso clínico: Importancia de la ecografía en los accesos vasculares difíciles. Diálisis y Trasplante. 2014; 35 (4):169-171.

3. Grau Pueyo C, Ibeas López J., Mateos Álvarez M. et al. Incorporación de enfermería a un equipo de gestión multidisciplinar del acceso vascular nativo. XXXII Congreso SEDEN. 2007.

4. López González A., Fernández Rivera C., Díaz Rodríguez L., Ornosa Agra MC. Uso de la ecografía como apoyo de la técnica de redireccionamiento de la aguja en el dolor de la fístula arteriovenosa durante la hemodiálisis. Enfermería Nefrológica, vol. 18, núm. 1, enero-marzo, 2015, pp. 58-60.

5. Granados Navarrete I., Grau Pueyo C, Ibeas López J, et al. Ecografía portátil solicitada por enfermería como herramienta complementaria en la monitorización del acceso vascular para hemodiálisis: consolidación de una técnica. XXXIII Congreso Nacional SEDEN. 1996.

6. Hernández López J. Punción con ecografía dirigida de la fístula arteriovenosa dificultosa. Diálisis y transplante. 2014; 35(1): 39-42.

7. Martín-Navarro J., Gutiérrez-Sánchez MJ, PetkovStoyanov V. Ecografía seriada del acceso vascular. Nefrología. 2012;32:116-8.

8. Pons Calvo C., Vinuesa García-Ciaño X., Grau Pueyo C. Dificultad de punción como indicador de patología oculta del acceso vascular para hemodiálisis: papel de enfermería en la solicitud y confirmación ecográfica. Enfermería Nefrológica 2012; 15 Suppl (1): 17/83.

9. Hernández López J., Rodríguez González M, González Ríos C, Mayor Iturburuaga J.M. Papel de la autoayuda con la ecografía en los accesos vasculares para hemodiálisis en la enfermería nefrológica. Diálisis y Trasplante .2015;36: 59-60. http://dx.doi.org/10.1016/j.dialis.2015.04.029

9. Moreno SánchezT, et al.Valor de la ecografía doppler en la disfunción de los accesos vasculares periféricos para hemodiálisis. Radiología. 2014;56(5):420428. http://dx.doi.org/10.1016/j.rx.2012.12.005 